\title{
LONGITUDINAL BEAM PARAMETERS STUDY IN THE SNS LINAC*
}

\author{
A. V. Feschenko, L. V. Kravchuk, A. A. Menshov, INR, Moscow, Russia \\ Alexander V. Aleksandrov, Saeed Assadi, John Galambos, Stuart Henderson, ORNL, Oak Ridge, \\ Tennessee
}

\section{Abstract}

SNS Linac utilizes several accelerating structures operating at two frequencies. CCL and SCL operate at $805 \mathrm{MHz}$ while $402.5 \mathrm{MHz}$ is used for RFQ and DTL. Beam transfer from the previous part of the accelerator to the subsequent one requires careful longitudinal matching to improve beam transmission and to minimize beam losses. Longitudinal beam parameters have been investigated with the help of three Bunch Shape Monitors installed in the inter-segments of the first CCL Module. The results of bunch shape observations for different accelerator settings are presented. Longitudinal beam emittance has been measured and optimized. Longitudinal beam halo has been evaluated as well.

\section{INTRODUCTION}

The SNS linac consists of an $\mathrm{H}^{-}$injector, capable of producing one-ms-long pulses with $38 \mathrm{~mA}$ peak current, chopped with a $68 \%$ beam-on duty factor and repetition rate of $60 \mathrm{~Hz}$ to produce $1.6 \mathrm{~mA}$ average current, an $86 \mathrm{MeV}$ Drift Tube Linac (DTL), a $185 \mathrm{MeV}$ Coupled Cavity Linac (CCL), a $1 \mathrm{GeV}$ Super Conducting Linac $(\mathrm{SCL})$ and associated transport lines [1].

Currently, there are two types of diagnostics in use for longitudinal bunch profile measurements. A Laser Bunch Shape Monitor (LBSM) is installed in the MEBT and three Bunch Shape Monitors (BSMs) are installed in the first module of the CCL.

In the LBSM a train of short pulses of light from a mode-locked laser synchronized with the $5^{\text {th }}$ subharmonic of $402.5 \mathrm{MHz}$ SNS beam frequency strips one electron from the negative hydrogen ions. The number of liberated electrons collected by the detector is proportional to the number of the ions in the volume of photons and ions interaction region. By scanning the laser phase relative to the bunch phase the longitudinal bunch profile is measured.

The BSM principle is based on coherent transformation of the ion bunch temporal structure into temporal structure of secondary electrons emitted from a thin wire, inserted into the beam, which is analyzed using transverse deflection in an RF deflector synchronized with the RF frequency of the linac. Details of the BSM design and its characteristics can be found in [2]. The SNS BSM has an additional energy selecting magnet which separates low energy secondary electrons from relatively high energy stripped electrons and thus increase the dynamic range of the measurements. We plan to install an additional BSM at the CCL exit and two BSMs at the SCL exit in near future.

*SNS is managed by UT-Battelle, LLC, under contract DE-AC0500OR22725 for the U.S. Department of Energy

04 Hadron Accelerators

\section{EXPERIMENTAL RESULTS}

\section{LBSM Measurements in the MEBT}

Typical bunch profiles measured by the LBSM in the MEBT are shown in Fig. 1. The bottom right plot in Fig. 1 shows the measured dependence of the RMS bunch width on the buncher phase. Good agreement with the PARMILA simulation confirms normal operation of the RFQ and proper tuning of the MEBT longitudinal focusing.
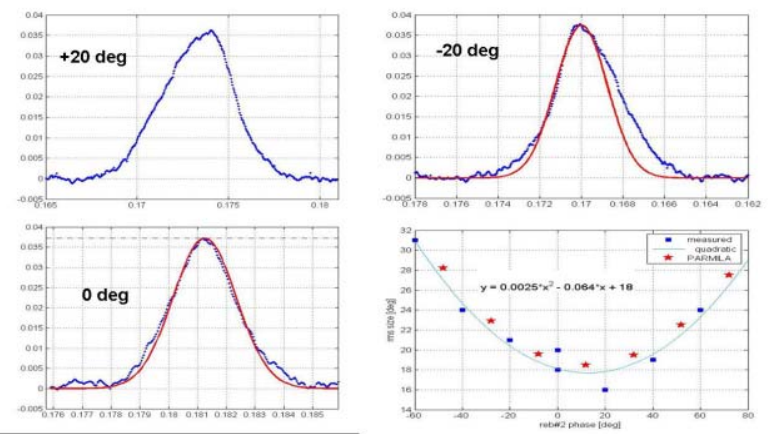

Figure 1: Bunch profile measurements in the SNS MEBT. Plots on top are for the buncher phase shifted from the nominal set point. Bottom left is for the nominal buncher setting. Bottom right is comparison of RMS bunch width vs. buncher phase dependence with the PARMILA simulation.

\section{Longitudinal Profile Measurements in the CCL}

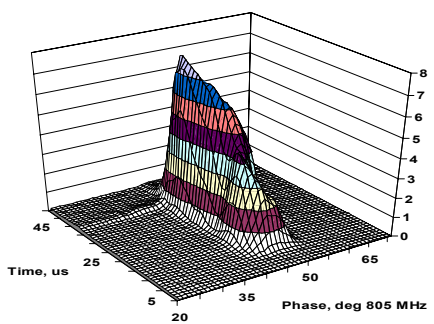

Figure 2: Typical data for nominal accelerator set point.
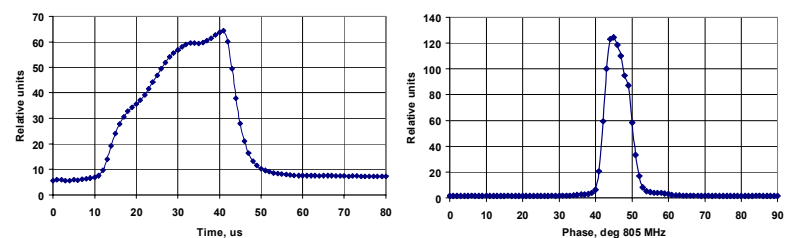

Figure 3: Secondary electron current from the target and the bunch shape. 
A typical longitudinal charge distribution in the bunch along the beam pulse measured by the BSM in the CCL is shown in Fig2. By integrating over the selected time interval within the pulse one obtains bunch profile during this time interval as shown in Fig. 3 right. By integrating over full phase interval one obtains beam pulse shape as shown in Fig. 3 left. Observing the bunch shape evolution along the pulse provides valuable information for debugging RF system problems such as improper tuning of the feedback system. An example is shown in Fig.4 where the bunch phase variation along the pulse is shown for the cases of good (on the right) and poor (on the left) beam loading compensation.
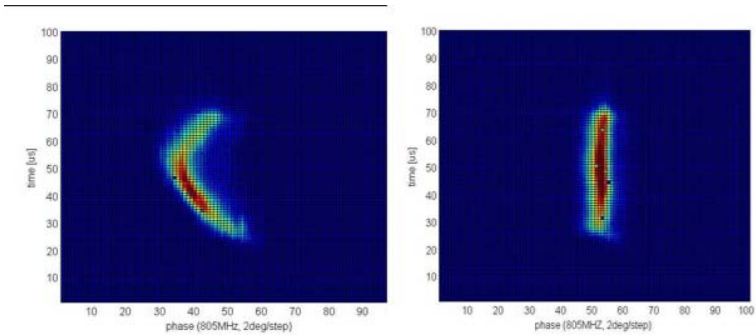

Figure 4: Longitudinal bunch shape along the bunch in case of poor (left) and good (right) beam loading compensation by the LLRF system.

Another example is shown in Fig.5, where measured longitudinal bunch profiles are shown for the cases of the correct setting of the buncher phase in the MEBT and for the phase shifted by 180 degrees. In the second case the buncher defocused particles longitudinally filling the whole longitudinal acceptance of the linac. Surprisingly, we observed significant losses not in the DTL to CCL transition area as one would expect but much farther downstream in the HEBT. Measuring the bunch profile with the BSM helped to identify cause of the problem quickly.

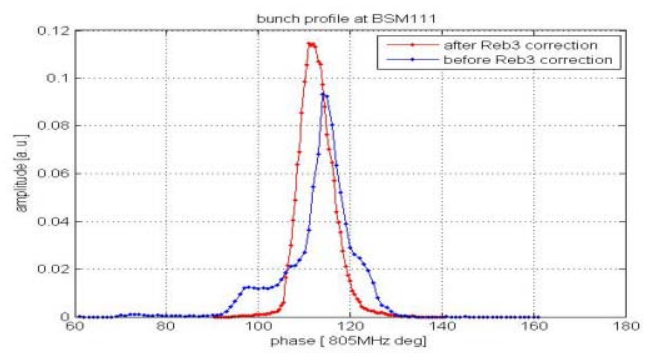

Figure 5: Longitudinal bunch profiles for the cases of the nominal buncher phase (red) and shifted by $180^{\circ}$ (blue).
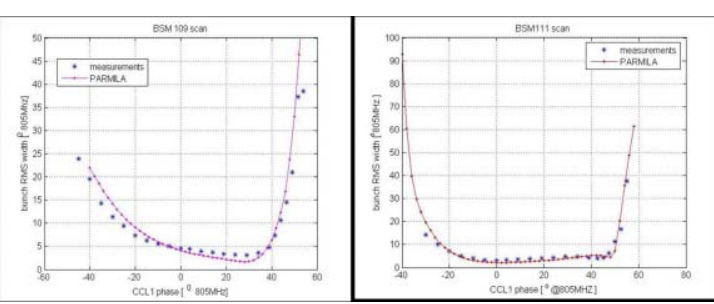

Figure 6: Comparison of the measured dependence of the longitudinal RMS bunch size on CCL1 RF phase with PARMILA simulations at two locations.
Longitudinal bunch size measurements are very useful for validating linac beam dynamics simulation model as shown by example in Fig. 6, where comparison of the measured and simulated bunch RMS size dependence on CCL1 RF phase is presented.

\section{Longitudinal Emittance Measurements}

Equivalent Twiss parameters of the longitudinal distribution can be calculated if longitudinal beam size is measured at three locations and transport matrix between these locations is known. Accuracy of the calculated parameters was increased by measuring beam sizes at different RF field amplitudes of $0.925 \mathrm{E}_{0}, 0.950 \mathrm{E}_{0}$, $0.975 \mathrm{E}_{0}, 1.000 \mathrm{E}_{0}, 1.025 \mathrm{E}_{0}, 1.050 \mathrm{E}_{0}$ и $1.075 \mathrm{E}_{0}$ with the corresponding correction of the bunch phase. Experimental data were cut below threshold of $1 \%, 3 \%$, $5 \%$ or $10 \%$. Beam size was defined as full width at the threshold level or statistical RMS size. Transport matrixes were calculated using PARMILA including the space charge effect. In principle, the transport matrixes should be found in iterations but a single iteration was sufficient in our case because the measured parameters were close to the design values. The straight lines corresponding to bunch boundaries measured at three locations at different RF field amplitudes and transformed to the CCL entrance are shown in Fig. 7. Parameters of the equivalent Twiss ellipse were found by minimizing RMS distance from the ellipse boundary to all 21 lines.

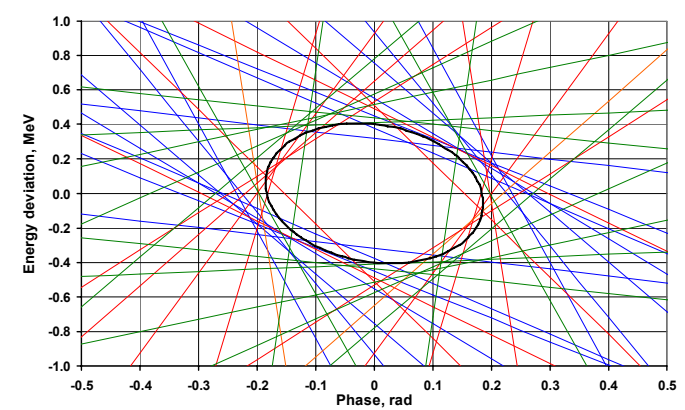

Figure 7: Bunch boundaries transformed to the entrance of CCL\#1 and an equivalent phase ellipse.

Equivalent phase ellipses calculated for different threshold levels are shown in Fig.8 for full width at the base bunch size definition and in Fig.9 for RMS bunch size definition.

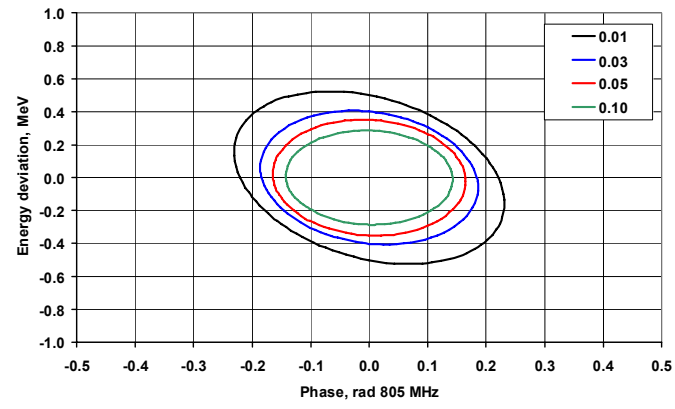

Figure 8: Equivalent phase space ellipses for different threshold levels. Full width at the base bunch size definition is used. 


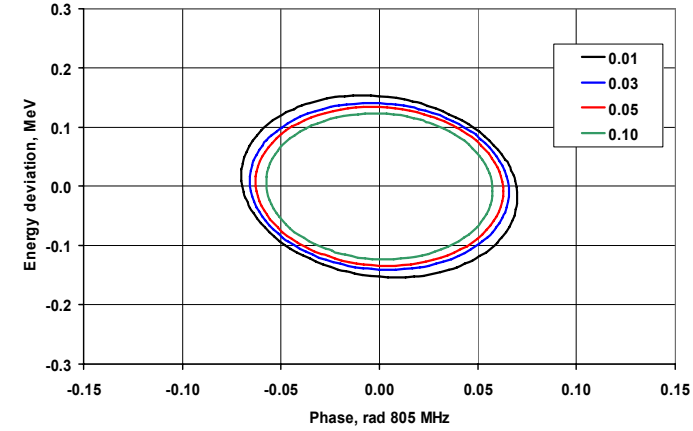

Figure 9: Equivalent phase space ellipses for different threshold levels. RMS bunch size definition is used.

It should be noted that the RMS bunch size definition gives more weight to particles in the bunch core compared to the full width of the base definition which is more affected by tail particles. As a result the dependence of ellipse orientation on threshold level is stronger in the latter case indicating non-linearity of longitudinal particles motion for large amplitude particles.

Twiss parameters of the equivalent ellipses measured at $20 \mathrm{~mA}$ beam peak current during the latest run are given in Table 1. The nominal design value of the longitudinal emittance is $0.006 \pi \cdot \mathrm{MeV}$ for the nominal beam peak current of $38 \mathrm{~mA}$. Typically we observe measured emittance variation from 1.2 to 2.0 times the nominal value from one run to another. The exact correlation of its value with different linac parameters remains to be established.

Table 1: Phase Ellipse Parameters

\begin{tabular}{|c|c|c|c|c|c|c|c|c|}
\hline \multirow{2}{*}{$\begin{array}{c}\text { Cutoff } \\
\text { Level }\end{array}$} & \multicolumn{4}{|c|}{ Base } & \multicolumn{4}{c|}{ RMS } \\
\cline { 2 - 9 } & $\alpha$ & $\begin{array}{c}\beta \\
\mathrm{eVV}^{-1}\end{array}$ & $\begin{array}{c}\gamma \\
\mathrm{MeV}\end{array}$ & $\begin{array}{c}\varepsilon \\
\mathrm{MeV}\end{array}$ & $\alpha$ & $\begin{array}{c}\beta \\
\mathrm{MeV}^{-1}\end{array}$ & $\begin{array}{c}\gamma \\
\mathrm{MeV}\end{array}$ & $\begin{array}{c}\varepsilon \\
\mathrm{MeV}\end{array}$ \\
\hline 0.01 & 0.3067 & 0.4605 & 2.3760 & 0.1158 & 0.1204 & 0.4595 & 2.2080 & 0.0107 \\
\hline 0.03 & 0.1534 & 0.4636 & 2.2080 & 0.0745 & 0.0697 & 0.4672 & 2.1510 & 0.0092 \\
\hline 0.05 & 0.0654 & 0.4689 & 2.1420 & 0.0578 & 0.0613 & 0.4686 & 2.1420 & 0.0084 \\
\hline 0.10 & 0.0429 & 0.4984 & 2.0100 & 0.0408 & 0.0516 & 0.4661 & 2.1510 & 0.0071 \\
\hline
\end{tabular}

\section{Observation of Longitudinal Tails and Halo}

The ability of measuring low level tail and halo in the longitudinal distribution could be very important for minimizing particles loss in the linac. Use of a secondary electron multiplier in the BSM design provides the possibility of varying detector gain over a wide range. Performing measurements of the same distribution with different gains and stitching the pieces together allows obtaining bunch profile with up to 5 decades of dynamic range as illustrated in Figs. 10 and 11.

The integrated density distribution function showing fraction of particles outside of a phase range is presented in Fig. 12. There is a monotonic exponential reduction of charge density from the bunch center to the boundary of the acceptance at about 40 degrees. We have not studied in detail yet the effect of various accelerator parameters on the level of the tail of the distribution but see it as a great potential for reducing beam loss.
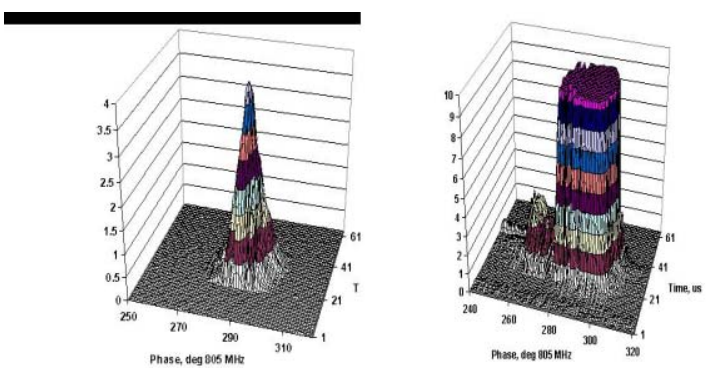

Figure 10: BSM measurements with the detector gain $\mathrm{g}=1$ (left) and g=160 (right).

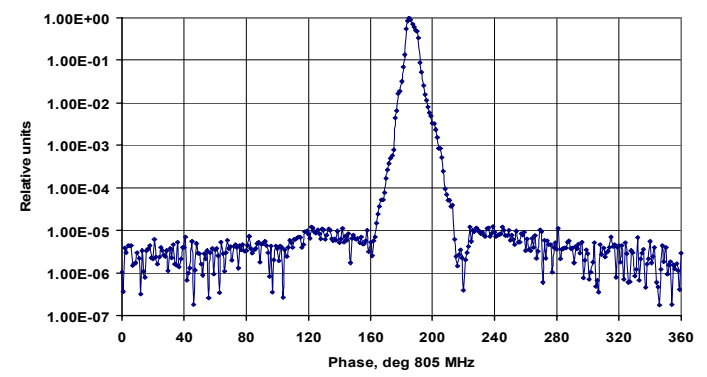

Figure 11: Longitudinal bunch profile combined from several measurements with gain ranging from 1 to 3500 .

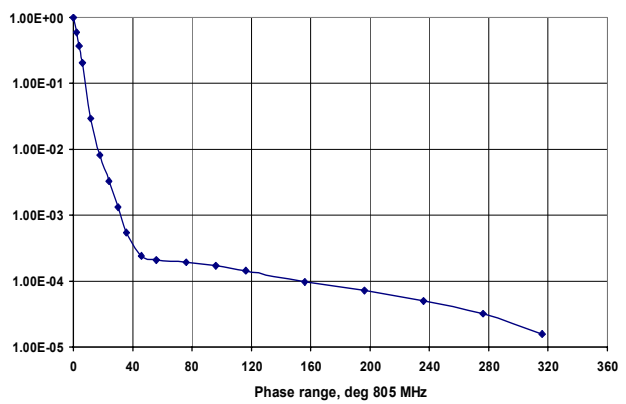

Figure 12: Fraction of particles outside of a given phase interval.

\section{CONCLUSIONS}

Study of the longitudinal beam parameters provides valuable information for troubleshooting and tuning the SNS linac, validation of the beam dynamics simulation models. The Beam Shape Monitors with energy selecting magnet are capable of measuring low level tails in the longitudinal distribution making possible experimental study of the halo. We expect to continue this study with the goal of reducing beam losses throughout the SNS accelerator and better understanding of beam dynamics in the linac.

\section{REFERENCES}

[1] S. Henderson. "Status of the Spallation Neutron Source: Machine and Science", these proceedings.

[2] A.Feschenko. PAC2001, Chicago, June 2001, V. 1, p. 517.

[3] A. Mirzojan et al. Voprosy Atomnoi Nauki i Tekhniki. V. 4,5 (31,32), Kharkov, 1997, p. 92 (in Russian). 